\title{
THE USE OF TOFACITINIB IN THE TREATMENT OF IMMUNE-MEDIATED UNIVERSAL ALOPECIA IN A PATIENT WITH SYSTEMIC LUPUS ERYTHEMATOSUS: A CASE REPORT
}

Sanna Paula Pires Mariano, ${ }^{1, \star}$, Thyago Talles de Almeida Santana ${ }^{1}$, Daniel Freire de Medeiros ${ }^{1}$, Danielle Christinne Soares Egypto de Brito ${ }^{1}$, Ana Karla Guedes de Melo $^{1}$, Eutilia Andrade Medeiros Freire ${ }^{1}$

1.Universidade Federal da Paraíba, João Pessoa (PB), Brazil.

*Corresponding author: sanninhapaula@hotmail.com

\section{BACKGROUND}

Systemic lupus erythematosus (SLE) is an autoimmune disease with heterogeneous clinical presentation. The biologic pathways that lead to loss of tolerance in SLE includes multiple autoreactive cell types (B, T, dendritic, Th17 and regulatory T cells) and abnormal cytokine milieus, genetic factors, environmental and hormonal influences. Tofacitinib is an orally administered Janus kinase inhibitor that has been approved for the treatment of moderate to severe rheumatoid arthritis and psoriatic arthritis. Many of the inflammatory cytokines implicated in the pathogenesis of SLE signal via the JAK-STAT pathways. Janus kinase inhibitors have been found to have efficacy in various murine models of lupus.

\section{CASE REPORT}

A 31-year-old female patient with SLE for six years presented with cutaneous, joint and hematological involvement. In the middle of 2017, she developed universal alopecia and exacerbation of polyarthritis that did not respond to the usual therapeutics, with an increase of corticotherapy, hydroxychloroquine, methotrexate, azathioprine and dapsone. Although, the administration of $5 \mathrm{mg}$ of tofacitinib twice a day in addition to the administration of hydroxychloroquine was initiated, resulting in total improvement of the arthritis and partial improvement of the alopecia in four months. Additionally, there was significant pilification of the eyebrows, eyelashes, axillary hair and pubic hair. Adverse events related to tofacitinib were not observed.

\section{CONCLUSION}

Targeting JAKs is an attractive therapeutic possibility for SLE. In this clinical case, tofacitinib had initial fast action between two and four weeks. Moreover, it has promoted meaningful hair growth in the patient with immune-mediated universal alopecia, sparing corticosteroids and presenting a great safety profile. Nevertheless, more extensive studies are needed in order to make these findings reproducible. 